\title{
THE HUBBLE CONSTANT AND THE DECELERATION PARAMETER
}

\author{
G. A. TAMMANN \\ Astronomisches Institut der Universität Basel, Binningen, Switzerland \\ and \\ Hamburger Sternwarte, Hamburg-Bergedorf, F.R.G.
}

\begin{abstract}
A preliminary report is given of recent work with A. Sandage on the Hubble constant. Through a chain of distance indicators in Sc and Ir galaxies (cepheids, brightest stars, $\mathrm{H}$ II regions, and luminosity classes) the distance scale is carried beyond any possible local anisotropy of the velocity field. Special care is taken to allow for the dependence of the intrinsic properties of the distance indicators on the size of the parent galaxy, and for the effect of the Malmquist correction. $H_{0}$ is found to be $55 \pm 7 \mathrm{~km} \mathrm{~s}^{-1} \mathrm{Mpc}^{-1}$; within the errors no systematic changes with distance were found.

A formal value of the deceleration constant $q_{0}=1 \pm 1$ was recently derived by Sandage (1972a) and Sandage and Hardy (1973). The most important correction to this value is probably the luminosity evolution of galaxies, which tends to push $q_{0}$ below 0.5 . The ensuing evidence for an open universe is also favored by independent arguments.
\end{abstract}

The expansion parameter and the deceleration parameter play a key rôle in all cosmologies. Moreover, all zero-pressure Einstein-Friedmann models with zero cosmological constant are fully defined by the observable, present values of these parameters: the Hubble constant $H_{0}$ and the deceleration constant $q_{0}$. The compatibility of the body of known observations with some Friedmann models as well as the simplicity of these models justify considering $H_{0}$ and $q_{0}$ the fundamental numerical constants of cosmology. Part of their fascination lies in the fact that they connect the 'time scale of creation' (Sandage, 1968a) and the eschatology of the Universe with empiricism.

In addition the Hubble constant is a useful tool for the distance determination of galaxies with known redshifts for an intermediate distance range - and this is also true out to the observational limits if allowance is made for the value of $q_{0}$.

This may explain why so much work has gone into the determination of the numerical values of $H_{0}$ and $q_{0}$.

\section{The Hubble Constant}

In recent years there has been a considerable number of papers on the value of $H_{0}$ (e.g. Holmberg, 1964; Sandage, 1968b; Heidmann, 1970; Roberts, 1972; de Vaucouleurs, 1972) and also several review papers (Tammann, 1969; van den Bergh, 1970, 1972; Heidmann, 1972). Therefore we will not attempt to repeat here all the arguments. Instead an account is given of some recent work by Sandage and the author. The discussion of the data is not quite completed yet, and the following remarks have still the character of being preliminary. The definitive version will be published elsewhere (Sandage and Tammann, 1974a, b), and it is this latter version which should be consulted in the future.

The essential problem of the determination of the Hubble constant $H_{0}$ had been realized by Hubble in 1936: the distance scale has to be forced out to distances where 
the cosmological expansion components are large compared to the random motions of individual galaxies. Since the random motions may be of the order of $\lesssim 300 \mathrm{~km} \mathrm{~s}^{-1}$ for field galaxies, good distances have to be obtained for galaxies with recession velocities $\gtrsim 1000 \mathrm{~km} \mathrm{~s}^{-1}$; that corresponds roughly to the distance of the Virgo cluster.

Hubble had revised his original value of $H_{0}=500$ (1929) to 550 (Hubble and Humason, 1931), and settled then for $530 \mathrm{~km} \mathrm{~s}^{-1} \mathrm{Mpc}^{-1}$ (Hubble, 1936). These small changes indicate that Hubble believed the uncertainty of his determination to be quite small. However, three major, systematic corrections to his distance scale have become necessary during the last four decades, one concerning his zero point and two being stretch factors to his scale:

(1) Baade (1952) showed that the zero point of the original period-luminosity relation for cepheid variables was too faint, and that all extragalactic distances had to be increased by a factor of 2 . His conclusion was based on the faintness of Population II stars in M31 and on Sandage's (1953) color-magnitude diagram for M3. Improved photometry of the cepheids in M31 (Baade and Swope, 1955, 1962) actually increased its distance by a factor of 3.7 .

(2) Beyond the range of cepheids (the present observational limit lies at $(m-M) \lesssim$ $\lesssim 28^{m}$, corresponding to $d \lesssim 3.5 \mathrm{Mpc}$ or $v_{0} \lesssim 200 \mathrm{~km} \mathrm{~s}^{-1}$ ) Hubble's distance scale relied heavily on the brightest stars in spiral galaxies. The corresponding distances were too small for two reasons:

(a) Hubble's magnitude scale, based on and extrapolated from Seares' (Seares et al., 1930) magnitudes, was too bright at the faint end. This was first suspected by Baade (cf. e.g. Baade, 1963) and finally confirmed by photoelectric photometry (Stebbins et al., 1950). A definitive photoelectric scale down to $22^{m}$ became available in 1952 through the work of W. A. Baum in SA 68, Baade's comparison field for M31.

(b) Hubble had misidentified very bright $\mathrm{H}$ II-regions in external galaxies as very bright stars, which led him to underestimate their distances. The error was first noted by Humason et al. (1956) on red plates, and was definitely corrected by Sandage (1958).

After correcting for (a) and (b) the evidence seemed to favour values of $H_{0}$ near 75 (Sandage, 1958, 1962).

(3) Only slowly has it been realized that some fundamental distance indicators change their properties not only with the type of the parent galaxy, but also - within a given type - with its luminosity, in the sense that the distance indicators are larger and/or more luminous in larger galaxies. One might expect that this effect averages out, - however, the nearer calibrating late-type galaxies (those for which cepheid distances are available) are all of small or moderate size, whereas the distant galaxies considered tend to be giants or supergiants. So far the effect has been established for three distance indicators:

(a) Brightest Stars. Hubble and Humason (1931) had anticipated the luminosity dependence of the brightest stars on the size of the parent galaxy, but they failed to detect the effect due to too small a number of independent distances. In his pioneering paper on nearby groups of galaxies Holmberg (1950) succeeded in demonstrating the existence of the effect, but he had still to rely on quite heterogeneous photometric 
data. A definite dependence of the brightness of the brightest blue stars on the brightness of the parent galaxy has been established recently on the basis of improved photometry in all spiral and irregular galaxies with known cepheid distances (Sandage and Tammann, 1974b). The brightest blue stars reach $M_{B}=-7^{m} .5$ in dwarf irregulars and at least $-10^{m}$ in the largest supergiant spirals. This correlation can be understood as a first approximation as the statistical effect of samples of different size, all drawn from a unique luminosity function.

(b) H II regions. Similarly, - and perhaps as a direct consequence of the sampledependent qualities of the brightest blue stars, - the diameters of the largest $\mathrm{H}$ II regions in irregular and spiral galaxies depend on the luminosity of the parent galaxy, as revealed by galaxies with known cepheid distances (Sandage and Tammann, 1974a). The interpretation of this dependence being a statistical effect is also supported by Hodge's (1967) result, who found that the population size of $\mathrm{H}$ II regions in Sc galaxies is proportional to the intrinsic galaxian luminosity. It was not possible to detect this effect in Sérsic's (1960) early investigation, because at that time no $\mathrm{H} \alpha$ plates were available and the $\mathrm{H}$ II-regions had to be measured on blue plates.

Neglecting this effect and using only nearby galaxies of moderate size as calibrators (LMC, SMC, and M33) leads to an H II distance of M101 (a supergiant spiral) which is too small by a factor of 2 .

For the distance determination the size dependence of $\mathrm{H}$ II regions on the luminosity of the parent galaxy is unfortunate, because the galaxian luminosity and the expected linear size of the largest $\mathrm{H}$ II regions are not known as long as the distance is undetermined. An equivalent dependence of the $\mathrm{H}$ II region size on van den Bergh's (1960) distance-independent luminosity classes has proved a powerful instrument in overcoming this difficulty.

(c) Globular Clusters. Comparing the brightest globular clusters in the Galaxy and in M31 with the brightest cluster in M87, an elliptical Virgo cluster member, Sandage $(1968 \mathrm{~b})$ derived $H_{0} \simeq 75$; he commented that this value would be lower if the globular clusters in M87 proved to be exceptionally luminous. This could be expected from the extremely large cluster population of M87 (Racine, 1968), and in fact Racine (1970) found that the globulars in other Virgo cluster galaxies are considerably fainter. A positive correlation between maximum cluster luminosity and luminosity of the parent galaxy was found also by de Vaucouleurs (1970), although using a mixtmre of open clusters and globular clusters; he could explain the effect as a statistical consequence of the sample size, i.e. the luminosity of the parent galaxy.

There can be little doubt that some additional distance indicators, including some which involve integral properties of galaxies, depend on the galaxy size to an extent which cannot be neglected.

Explicitly allowing for the effect described in paragraph (3) we have now attempted to extend the distance scale to distances of the order of $100 \mathrm{Mpc}$, i.e. beyond the influence of any possible non-linearity or anisotropy of the Hubble flow. The procedure follows the following steps (compare Sandage and Tammann, 1971b; Sandage, 1971b, 1972b): 
(1) The fundamental distance indicators are classical cepheids. After a recalibration of the period-luminosity-third parameter relation (Sandage and Tammann, 1968, 1969,1971 c) distances can be derived for five late-type galaxies in the Local Group with known cepheids [LMC, SMC (Gascoigne, 1969); M33 (Hubble, 1926); NGC 6822 (Kayser, 1967); and IC 1613 (Sandage, 1971a)]. The cepheid distance of NGC 2403 (Tammann and Sandage, 1968), a member of the M81 group, yields distances for five additional late-type galaxies (NGC 2366, NGC 4236, IC 2574, Ho II, and Ho I), since it can be shown independently that the members of the M81 group lie at a common distance. These eleven Sc or Ir I galaxies are used as the fundamental calibrators; their moduli are $(m-M)<28^{m}$.-

It should be noted that cepheids are tied into the Population I distance scale, and depend therefore directly on the adopted distance of the Hyades. It seems now that van Bueren's (1952) moving-cluster distance is in fact reliable at a level of about $5 \%$ (Upton, 1970 and references therein, 1971).

It has been suspected that certain statistical differences of cepheids in the Galaxy and in SMC are due to chemical differences, which shift the instability strip in the $M_{V}-(B-V)$-plane. It is needless to say that this would question the value of cepheids as distance indicators. However, it has been possible to explain the observed differences with the assumption of a unique instability strip, which is differently populated in the Galaxy and in SMC (Sandage and Tammann, 1971c). As originally proposed by Christy (1970) the different populations could well be understood by assuming that the evolutionary loops during the red-giant phase, which feed the instability strip, are somewhat different for the two galaxies; the form of these loops is in fact extremely sensitive to various parameters (for a more thorough discussion of the problem see Sandage, 1972b, cf. also Lauterborn et al., 1971). But there is no definite proof yet for the uniqueness of the instability strip and the question arises, why is it necessary to rely exclusively on cepheids?

The answer to the question is that cepheids offer the tremendous advantage of having an exceptionally small intrinsic scatter of $\sigma \leqslant 0^{m} \cdot 1$ about the mean periodluminosity - color (or amplitude) relation for an observed period and color (or amplitude) (Sandage and Tammann, 1969, 1971c). If one works to the telescope limit, and for the extragalactic distance scale one almost always does, - the discrimination against intrinsically fainter objects can become very severe. Accordingly the $\mathrm{Malm}$ quist correction (Eddington, 1914; Malmquist, 1921) has to be considered, which amounts under idealized conditions to $\Delta M=1.38 \sigma^{2}$. This introduces a systematic error always in the sense that distances are underestimated, - but for cepheids the effect can be totally neglected, since it amounts to only $\Delta M \simeq 0^{m} 01$. It is clear that other distance indicators with $\sigma$, let us say, $0^{m} \cdot 3$ or $1^{m} \cdot 0$ have 10 times or even 100 times larger systematic errors. A correction for these errors requires quite good control of the statistical sample concerning completeness to a given magnitude limit as well as size and distribution of the intrinsic scatter. These conditions are normally not fulfilled. This may indicate therefore that low weight should be given at present to other primary distance indicators like RR Lyrae stars and spectral luminosity in- 
dicators, which eventually will yield a very important check on the cepheid distances.

(2) The eleven calibrating galaxies yield the relation between the linear size of the largest $\mathrm{H}$ II regions (as measured on $\mathrm{H} \alpha$ plates) and the luminosity class of the parent galaxy (Sandage and Tammann, 1974a).

(3) The dependence of the brightness of the brightest blue stars on the absolute magnitude of the parent galaxy is established using again the calibrating galaxies.

(4) The distance of M101, the nearest supergiant Sc galaxy (ScI), is derived by six different methods (including two methods via step 2 and 3). The absence of cepheids puts a stringent lower limit to the distance (Sandage and Tammann, 1971a). The distance determination is greatly facilitated because M101 has five late-type, bona fide companion galaxies, whose size is well bracketed by the calibrating galaxies. The resulting modulus for the M101 group is $(m-M)^{0}=29^{m} \cdot 3 \pm 0^{m} \cdot 3$; the corresponding distance is larger by a factor of 2 than the formerly adopted, provisional value (Sandage, 1962).

The great importance of the M101 distance lies in the fact that it allows the extension of the calibrations in step 2 and 3 out to the largest spirals. In this way the brightness dependence of the brightest stars and the size dependence of the largest $\mathrm{H}$ II regions are defined for the full range, from dwarf galaxies (IC 1613, Ho I) to supergiant $\mathrm{Sc}$ I galaxies (M101).

(5) The size of the largest $\mathrm{H}$ II regions are measured on $\mathrm{H} \alpha$ plates in about 40 latetype field galaxies with known luminosity class. Individual distances are derived via step 2; the resulting distance moduli are all $(m-M)^{\circ}<32^{m}$.

(6) The mean absolute magnitudes for different luminosity classes (particularly for Sc I's) are derived, using galaxy distances from steps 1,4 , and 5 . The magnitudes are defined in Holmberg's (1958) $m_{p g}$-system, and they are corrected for galactic absorption (Sandage, 1973) and for inclination (Holmberg, 1958).

(7) A new sample of more than 50 distant Sc I galaxies is selected with $13^{m}<m_{p g} \lesssim$ $\lesssim 15^{m} .5$ and their redshifts are determined $\left(c z>5000 \mathrm{~km} \mathrm{~s}^{-1}\right)$.

(8) The combination of apparent magnitudes and velocities from step 7 with absolute magnitudes from step 6 leads to distance moduli $\left(35^{m}<m-M<38^{m}\right)$ and, after correction for the Malmquist effect, - to a mean value of $H_{0}$ beyond any possible local disturbance of the velocity field.

The result for $H_{0}$ is $55 \pm 7 \mathrm{~km} \mathrm{~s}^{-1} \mathrm{Mpc}^{-1}$ (Sandage and Tammann, 1971b; Sandage, 1972c). Within the error range the result is the same for galaxies inside the Local Supercluster $\left(m-M<32^{m}\right)$, for the Virgo cluster $\left(m-M=31^{m} .5\right)$, and for the distant sample of Sc I galaxies $\left(m-M>35^{m}\right)$. For most practical purposes a value of $H_{0}=50$ is apparently a sufficient and convenient approximation.

The constancy of $H_{0}$ infringes on the possible non-linearity and/or anisotropy of the expansion rate within the Local Supercluster (de Vaucouleurs, 1971, and references therein). The present results on $H_{0}$ do not require such an effect, but they cannot exclude the possibility that small effects of this kind exist, because the detailed mapping of the local kinematic field is still not sufficiently advanced. This mapping demands very homogeneous observational data over the whole sky and an exact control 
of the statistical selection effects. For the cosmic value of $H_{0}$ the possibility of a local velocity perturbation is not really relevant, because the distant sample of Sc I galaxies lies at $c z>5000 \mathrm{~km} \mathrm{~s}^{-1}$, and Sandage's (1968c, 1972a; Sandage and Hardy, 1973) tight redshift-magnitude relation for the brightest cluster galaxies proves the nonexistence of large perturbations for $c z \gtrsim 3000 \mathrm{~km} \mathrm{~s}^{-1}$ (Sandage et al., 1972). A relatively small velocity anisotropy agrees qualitatively also with Partridge's (1974) reduction of the excess velocities originally inferred from the background radiation by Conklin (1969).

Support for $H_{0}$ values near $H_{0}=50$ has come recently from supernovae (Branch and Patchett, 1973; Kirshner, 1973). Also globular clusters support this value (Sandage, 1968b; de Vaucouleurs, 1970) if allowance is made for the exceptionally high brightness of globular clusters in giant galaxies. It should be stressed that these two routes are entirely independent of the present one, the supernovae depending on the purely physical Baade-Wesselink method and the globulars being tied into the Population II distance scale.

Unfortunately it would be an oversimplification to call on Abell and Eastmond's (1970) and Abell's (1972) value of $H_{0}=47$ for further support of the present value, because a severe difference exists between the two distance scales. Their value is derived from the Coma cluster, for which $\bar{v}_{0}=6866 \mathrm{~km} \mathrm{~s}^{-1}$ and $(m-M)^{\circ}=35^{m} .8$ is assumed. At the same time their corresponding distance modulus for the Virgo cluster is $(m-M)^{\circ}=31^{m} \cdot 1$, and if $\bar{v}_{0}$ (Virgo) $=1141 \mathrm{~km} \mathrm{~s}^{-1}$ (Tammann, 1972) it follows that $H_{0}=69$ at the distance of Virgo. The distance scale therefore implies a strong non-linearity of the expansion rate. The reason for this is that the adopted modulus difference Coma minus Virgo is $\Delta(m-M)^{\circ}=4 .^{m} 7$, whereas the mean velocity ratio of the two clusters requires only $\Delta(m-M)^{\circ}=3^{m} \cdot 9\left( \pm \sim 0^{m} 4\right)$ in the case of a constant expansion rate. Independent support of the latter value has come from the colors of E/S0 galaxies, which indicate $\Delta(m-M)^{\circ}=3^{m} \cdot 66 \pm 0^{m} \cdot 14$ (Sandage, 1972d). An additional check will eventually be provided by the apparent magnitude difference of supernovae of type I in the Virgo and Coma clusters; preliminary evidence, based on only three Coma supernovae with known maximum brightness, suggests $\Delta(m-M)^{\circ}$ $=3^{m} .9$ ( $\left.\pm \sim 0^{m} .3\right)$ (Branch, 1974; Yilmaz and Tammann, 1973) or according to data by Kowal (1968), even less.

It may be that other recent determinations of $H_{0}$, which led to values of 75 or even larger, are still affected by two systematic factors described above: (1) the variable properties of distance indicators with the type and size of the parent galaxy; and (2) the Malmquist correction. Either factor tends toward too small distances and too high values of $H_{0}$. Particularly, if one neglects the Malmquist effect, one comes to solutions which indicate $H_{0}$ to increase with distance; therefore results of this kind should be considered with scepticism.

There has been much comment on the steady decrease of the Hubble constant during the last four decades, and $H_{0}$ has been called jokingly the 'Hubble variable'. Why is it that the corrections have always led to a decrease of $H_{0}$ ? The principal reason is perhaps a very natural phenomenon; one is basically reluctant to accept the 
existence of objects which are much brighter and much larger than those one has previously experienced in the immediate neighborhood. Parallel cases are plentifully known in the history of astronomical development; an example is the magnitude calibration of spectral types of luminous stars. The possibility that the true value of $H_{0}$ is still smaller than 50 cannot be ruled out yet, but one may hope to have approached asymptotically the correct solution: from 1936 to $1958 H_{0}$ decreased from 530 to 75 , that is by a factor of 7 , as compared to a factor of only 1.4 during the last $15 \mathrm{yr}$.

One may wonder if it is possible to set a stringent lower limit to the value of $H_{0}$. This seems in fact very difficult. However, one should remember that the distance scale of the thirties raised two very severe problems: (1) the expansion time scale was too short to account even for geological ages; and (2) the Galaxy was an outstandingly large object as compared to other galaxies. These inconsistencies are now removed, the time scale being very satisfactory as discussed below, and the size of the Galaxy, - and also of M31, - fitting well into the morphological sequence of more distant galaxies. This twofold agreement would certainly be weakened if $H_{0}$ were appreciably lower than 50 .

\section{The Deceleration Constant $q_{0}$}

The possibilities and difficulties of determining $q_{0}$ from observations at great distances in general-relativity cosmologies with $\Lambda=0$ have been discussed very thoroughly in a well-known paper by Sandage (1961). This suggested that the most promising way to find $q_{0}$ is via the redshift-magnitude relation. The potential of this method has increased greatly as a result of the discovery that brightest cluster galaxies have impressively uniform luminosities and, hence, that they can be used as reliable standard candles to fathom the Universe. Through the photometric efforts of Westerlund and Wall (1969), Peterson (1970) and especially Sandage (1972a), and through difficult redshift determinations at very faint levels by Minkowski (1960), Baum (1962), and Oke (1971) by far the largest body of observations have so far been obtained for this route.

The first historically important attempts to derive $q_{0}$ from the magnitude-redshift relation were made by Humason et al. (1956), Baum (1957, 1962) and Sandage (1961). These determinations favored slightly a closed universe; they contained corrections to the observed magnitudes to various degrees of refinement, but they all did specifically exclude the effect of luminosity evolution of galaxies (For a discussion up to 1970 of the correction effects see Peach (1972)). Also Sandage's (1972a) recent determination of $q_{0}$ explicitly disregards luminosity evolution; he found from the Hubble diagram for 39 first-ranked cluster galaxies with $z<0.5$, after correcting the galaxian apparent magnitudes for galactic absorption, aperture effects, and $K$-dimming, a formal value for the deceleration constant of $q_{0}=1 \pm 1$ ( $2 \sigma$ error). This value has not been significantly changed by allowing for a detectable luminosity dependence on the Morgan-Bautz type and a slight dependence (at a 98\% confidence level) on the population size of the different clusters of galaxies involved (Sandage and Hardy, 1973). 
This formal solution requires still three corrections; the first of which is to decrease the true value of $q_{0}$ as it turns out, the other two to increase it:

(1) The luminosity evolution of galaxies;

(2) The effect of intergalactic dimming if it can be established that such dimming is a general feature of intergalactic space. (Present indications for such dimming on the basis of galaxy or cluster distribution depend on the absence of even very slight systematic selection effects in existing catalogues.) The possible influence of electron scattering in an ionized medium has been calculated by Bahcall and May (1968) and of absorption from intergalactic dust by Romano (1973).

(3) If most of the mass in the Universe is concentrated in individual objects, as the existence of galaxies suggests, the light propagation is not the same as in a homogeneous Friedmann universe. Expressions have been calculated by Zel'dovich (1964) and more generally by Kantowski (1969) and Dyer and Roeder (1973), indicating that in a universe with voids between mass points the brightness of an object would be observed too faint, and consequently that the observed value of $q_{0}$ would be too low. (It should be noted that this correction also affects the angular diameterredshift relation. The apparent lack of curvature in the angular size - redshift distribution of double radio sources (Kellermann, 1972) could point toward the observability of the effect, although angular-size evolution may here be the dominant cause).

Recent evidence seems to indicate that effect (1) is the most important: Baldwin et al. (1973) have concluded primarily from the strength of the $\mathrm{CO}$ absorption band at $2.3 \mu \mathrm{m}$ that the light in the nuclei of two spiral galaxies (including M31) and in the peculiar galaxy NGC 5195 is dominated by giant stars, and with this they have apparently decided a long-standing question. At this very moment there is still a (slight) ambiguity as to whether or not the conclusion holds also for giant ellipticals and if it therefore affects $q_{0}$. Final evidence by direct observations of gE's can be expected soon. There are, however, already strong indications that the $\mathrm{CO}$ band will also be found in gE's, because their stellar population resembles that of the central regions of spirals like M31 in all other aspects, according to continuum scans (Oke and Sandage, 1968) and to line strengths (Morgan and Osterbrock, 1969; Spinrad et al., 1971). If the light of gE's is dominated by giants indeed, it is clear that the effect of luminosity evolution is very important and that these galaxies have been much brighter in the past. An analysis by Tinsley (1973) indicates that the correction to $q_{0}$ (obs) is $\Delta q_{0}>1$, in the sense which decreases the observed value. Combining this with Sandage's formal value favors small values of $q_{0}$ (corr), and therefore an open universe.

There are two additional hints for a low value of $q_{0}$. Somewhat unexpectedly they are derived from relatively nearby observations:

(1) Gott and Gunn (1971) and Gunn and Gott (1972) have shown that if clusters of galaxies grow from small density perturbations, the amount of hot gas in the Coma cluster, as inferred from X-ray observations, limits the intergalactic gaseous mass and requires (if it is excluded that most of the mass is in invisible, condensed bodies) $q_{0}<0.1$. However, their argument has been criticized by Field (1974). 
(2) Sandage et al. (1972) have pointed out that the well-known density excess of roughly a factor of 2 in the Local Supercluster, as first discussed by Shapley and Ames (1932) and Reiz (1941), has apparently a very small effect on the local expansion rate. This seems to require that the kinetic expansion energy $T$ and, of course, also the total energy $E$ are much larger than the gravitational potential energy $\Omega$, because the observable variation $\delta \Omega$ has an unobservable effect on $T=E+\Omega$. If that is the case the matter density must be very small, and so $q_{0}$ must be. The argument would be invalidated if only a fraction of the total mass exhibited the typical clumping in clusters, and if most of the mass were uniformly distributed in some invisible form.

It will hardly ever be possible to determine $q_{0}$ from the observable mass in the Universe, because ex definitione the information on the unobservable mass is insufficient, and, even more important, because for a unique relation between mass density and $q_{0}$ a matter-dominated Friedmann model must be assumed $a$ priori. A way of estimating the mass density in the Universe is via the mean volume emissivity in photographic light due to galaxies. The values for this quantity from different authors (Oort, 1958; van den Bergh, 1961; Kiang, 1961; Peebles, 1971) agree surprisingly well; after applying a weighted correction for internal absorption in spiral galaxies, $l_{0}=2 \times 10^{8} L_{\odot} \mathrm{Mpc}^{-3}$ is adopted here (this figure and the following ones are, if appropriate, reduced to $H_{0}=55$ ). Combining this with a value for the mean massto-light ratio leads to the mean mass density. Two different assumptions can be made concerning $\mathfrak{M} / L$ :

(1) $\mathfrak{M} / L=20$; this value may be the best compromise for the detectable luminous mass in an actual mixture of ellipticals and spirals in the general field. A value of $\mathfrak{M} / L=4$ (Roberts, 1969) is here assumed for spirals and $\mathfrak{M} / L=30$ for the mean of ellipticals in pairs (Smart, 1973). The resulting mean density of luminous matter is then $\varrho_{\text {lum }}=3 \times 10^{-31} \mathrm{~g} \mathrm{~cm}^{-3}$, a value which agrees within factors of 3 with independent determinations (Shane and Wirtanen, 1967; Holmberg, 1969; Noonan, 1971a). The critical Einstein-de Sitter density being

$$
\varrho_{c}=\frac{3 H_{0}^{2}}{8 \pi G} \simeq 6 \times 10^{-30} \mathrm{~g} \mathrm{~cm}^{-3},
$$

leads then (since the energy density in the present Universe is assumed to be dominated by non-relativistic matter) to

$$
q_{0}=\frac{\varrho_{\text {lum }}}{2 \varrho_{c}}=0.025 .
$$

This value of $q_{0}$, which is possibly still affected by observational errors of factors of about 2-3, but which is independent of $H_{0}$, represents an absolute lower limit for all Friedmann models.

(2) $\mathfrak{M} / L=175$ is needed from the virial theorem, if clusters like the Coma cluster are stable (Rood et al., 1972). Only a fraction of galaxies are in clusters as rich as Coma, but a similar mass-to-light ratio is required to stabilize groups of galaxies (Geller and Peebles, 1973), and therefore the adopted value seems in general to be 
required from a dynamical point of view. There is still hope that the unseen mass may reside in the galaxies themselves, for instance in form of faint stars in the outer regions (Searle, 1973); or/and in extremely massive gE galaxies (at least for aggregates containing such objects), because Smart (1973) found a pronounced increase of $\mathfrak{M} / L$ with the luminosity of elliptical galaxies. Alternatively, the assumption that the missing mass is in form of intergalactic, but intra-cluster matter is the only possibility for avoiding the highly improbable conclusion of clusters being short-lived phenomena. In any case the virial mass corresponds to a mean mass density of $\varrho_{\text {vir }}=2.6 \times 10^{-30}$ $\mathrm{g} \mathrm{cm}^{-3}$, which again is in very satisfactory agreement with parallel, at least partially independent determinations (Abell, 1965; Noonan, 1971b). This density suggests a lower limit of $q_{0}=0.2$, and this still favors an open universe.

The above conclusion concerning the minimum value of $q_{0}$ could be changed decisively, if much mass should be detected in intercluster space. For instance Oort $(1970 \mathrm{a}, \mathrm{b})$ has proposed that only one fifteenth of the total mass is presently condensed in galaxies. The excess mass, if confined to clusters and groups, could just be the missing amount to stabilize clusters; if, however, the virial mass should eventually be found within galaxies, the proposed factor would require a still much higher total density, i.e. $\varrho \simeq 4 \times 10^{-29} \mathrm{~g} \mathrm{~cm}^{-3}$. This would be definitely enough to close the Universe.

Concentrating here on direct, observational evidence suggests that $q_{0}=0.1-0.2$ may presently be the best compromise (cf. Sandage, 1972c).

It is customary to test the combined consequences of astronomical constants, which are derived from observations. $H_{0}=55 \pm 7\left[H_{0}^{-1}=(17.7 \pm 2) \times 10^{9} \mathrm{yr}\right]$ and $q_{0}=0.1-$ -0.2 require a Friedmann time, i.e. the time since the beginning of the expansion, of $t_{0}=(0.78-0.85) H_{0}^{-1}$ (Sandage, 1961) or $t_{0}=(13-16) \times 10^{9} \mathrm{yr}$. This may be compared with the age of globular clusters, the best determinations of which yield $(10 \pm 3) \times 10^{9} \mathrm{yr}$ (Sandage, 1970) and $14 \times 10^{9} \mathrm{yr}$ or somewhat less (Böhm-Vitense and Szkody, 1973), and may also be compared with the age of the elements of $(11.7 \pm 2) \times 10^{9} \mathrm{yr}$ (Fowler, 1972). It has been stressed (e.g. Sandage, 1968a) that the near coincidence of these values is most impressive, and that it is, to say the least, a very attractive feature of Friedmann cosmologies.

While it is hoped that the present value of $H_{0}$ may not be too far from the truth, the choice of $q_{0}$ is not free of speculation. Apparently an infinite, non-singular future is the fate of our Universe, but the final answer lies still in the future - a future which is hoped to be much nearer.

\section{Acknowledgements}

It is a pleasure to thank Dr A. Sandage for a most stimulating period of cooperation. Thanks are also due to Dr H. W. Babcock for hospitality at the Hale Observatories, and to Prof. D. Lynden-Bell for a summer at the Institute of Astronomy, Cambridge, where part of this paper was written. It is gratefully acknowledged that the work on the Hubble constant reported here was supported by the U.S. National Science Foundation during a period of two years and that partial financial support was successively granted by the Swiss National Science Foundation. 


\section{References}

Abell, G. O.: 1965, Ann. Rev. Astron. Astrophys. 3, 1.

Abell, G. O.: 1972, in D. S. Evans (ed.), 'External Galaxies and Quasi-Stellar Objects', IAU Symp. 44, 341.

Abell, G. O, and Eastmond, S. : 1970, Bull. Am. Astron. Soc. 2, 179.

Baade, W.: 1952, Trans. IAU 8, 397.

Baade, W.: 1963, in C. Payne-Gaposchkin (ed.), Evolution of Stars and Galaxies, Harvard Univ. Press, Cambridge, Mass., p. 38f.

Baade, W. and Swope, H. H. : 1955, Astron. J. 60, 151.

Baade, W. and Swope, H. H.: 1963, Astron. J. 68, 435.

Bahcall, J. N. and May, R. M.: 1968, Astrophys. J. 152, 37.

Baldwin, J. R., Danziger, I. J., Frogel, J. A., and Persson, S. E. : 1973, Astrophys. Letters 14, 1.

Baum, W. A.: 1957, Astron. J. 62, 6.

Baum, W. A.: 1962, in G. C. McVittie (ed.), 'Problems of Extra-Galactic Research', IAU Symp. 15, 390.

Bergh, S. van den: 1960, Publ. David Dunlap Obs. 2, 159.

Bergh, S. van den: 1961, Z. Astrophys. 53, 219.

Bergh, S. van den: 1970, Nature 225, 503.

Bergh, S. van den: 1972, in G. Cayrel de Strobel and A. M. Delplace (eds.), L'âge des étoiles, Observatoire de Paris-Meudon, Meudon, Chapter 40.

Böhm-Vitense, E. and Szkody, P.: 1973, Astrophys. J. 184, 211.

Branch, D.: 1974, in C. Batalli Cosmovici (ed.), International Conference on Supernovae, D. Reidel Publ. Co., Dordrecht, p. 209.

Branch, D. and Patchett, B.: 1973, Monthly Notices Roy. Astron. Soc. 161, 71.

Bueren, H. G. van: 1952, Bull. Astron. Inst. Neth. 11, 385.

Christy, R. F.: 1970, J. Roy. Astron. Soc. Can. 64, 8.

Conklin, E. K.: 1969, Nature 222, 971.

de Vaucouleurs, G.: 1970, Astrophys. J. 159, 435.

de Vaucouleurs, G.: 1971, Publ. Astron. Soc. Pacific 83, 113.

de Vaucouleurs, G.: 1972, in D. S. Evans (ed.), 'External Galaxies and Quasi-Stellar Objects', IAU Symp. 44, 353.

Dyer, C. C. and Roeder, R. C.: 1973, Astrophys. J. Letters 180, L31.

Eddington, A. S.: 1914, Stellar Movements and the Structure of the Universe, Macmillan, London, p. 172.

Field, G.: 1974, this volume, p. 13.

Fowler, W. A. : 1972, in F. Reines (ed.), Cosmology, Fusion and Other Matters, Adam Hilger Ltd., London, p. 67.

Gascoigne, S. C. B.: 1969, Monthly Notices Roy. Astron. Soc. 146, 1.

Geller, M. J. and Peebles, P. J. E.: 1973, Astrophys. J. 184, 329.

Gott, J. R. and Gunn, J. E. : 1971, Astrophys. J. Letters 169, L13.

Gunn, J. E. and Gott, J. R.: 1972, Astrophys. J. 176, 1.

Heidmann, J. : 1970, Compt, Rend. Acad. Sci. Paris 271B, 658.

Heidmann, J.: 1972, in G. Cayrel de Strobel and A. M. Delplace (eds.), L'âge des étoiles, Observatoire de Paris-Meudon, Meudon, Chapter 37.

Hodge, P. W.: 1967, An Atlas and Catalog of H II Regions in Galaxies, Astronomy Department, Univ. of Washington, Seattle.

Holmberg, E. : 1950, Medd. Lunds Obs., Ser. II, No. 128.

Holmberg, E. : 1958, Medd. Lunds Obs., Ser. II, No. 136.

Holmberg, E.: 1964, Arkiv Astron. 3, 387.

Holmberg, E.: 1969, Arkiv Astron. 5, 305.

Hubble, E.: 1926, Astrophys. J. 63, 236.

Hubble, E.: 1929, Proc. Nat. Acad. Sci. 15, 168, Mt. Wilson Comm., No. 105.

Hubble, E.: 1936, The Realm of the Nebulae, Yale Univ. Press, New Haven, p. 167f.

Hubble, E. and Humason, M. L.: 1931, Astrophys. J. 74, 43.

Humason, M. L., Mayall, N. U., and Sandage, A. R. : 1956, Astron. J. 61, 97.

Kantowski, R.: 1969, Astrophys. J. $155,89$.

Kayser, S. E. : 1967, Astron. J. 72, 134.

Kellermann, K. I.: 1972, Astron. J. 77, 531.

Kiang, T.: 1961, Monthly Notices Roy. Astron. Soc. 122, 263. 
Kirshner, R. P.: 1973, private communication.

Kowal, C. T.: 1968, Astron. J. 73, 1021.

Lauterborn, D., Refsdal, S., and Weigert, A.: 1971, Astron. Astrophys. 10, 97.

Malmquist, K. G. : 1920, Medd. Lunds Obs., Ser. II, No. 22.

Minkowski, R.: 1960, Astrophys. J. 132, 908.

Morgan, W. W. and Osterbrock, D. E.: 1969, Astron. J. 74, 515.

Noonan, T. W.: 1971a, Publ. Astron. Soc. Pacific 83, 31.

Noonan, T. W.: 1971b, Publ. Astron. Soc. Pacific 83, 37.

Oke, J. B. : 1971, Astrophys. J. 170, 193.

Oke, J. B. and Sandage, A. R.: 1968, Astrophys. J. 154, 21.

Oort, J. H.: 1958, in R. Stoops (ed.), La structure et l'évolution de l'univers, Inst. Internat. Phys. Solvay, Bruxelles, p. 163.

Oort, J. H.: 1970a, Astron. Astrophys. 7, 405.

Oort, J. H.: 1970b, Science 170, 1363.

Partridge, R. B.: 1974, this volume, p. 157.

Peach, J. V.: 1972, in D. S. Evans (ed.), 'External Galaxies and Quasi-Stellar Objects'. IAU Symp. 44, 314.

Peebles, P. J. E.: 1971, Physical Cosmology, Princeton Univ. Press, Princeton, p. 57.

Peterson, B. A.: 1970, Astron. J. 75, 695.

Racine, R.: 1968, J. Roy. Astron. Soc. Can. 62, 367.

Racine, R.: 1970, J. Roy. Astron. Soc. Can. 64, 257.

Reiz, A.: 1941, Ann. Obs. Lund No. 9.

Roberts, M. S.: 1969, Astron. J. 74, 859.

Roberts, M. S. : 1972, in D. S. Evans (ed.), 'External Galaxies and Quasi-Stellar Objects', IAU Symp. 44, 12.

Romano, G. : 1973, Mem. Soc. Astron. Ital., N.S. 44, 47.

Rood, H. J., Page, T. L., Kintner, E. C., and King, I. R.: 1972, Astrophys. J. 175, 627.

Sandage, A. R.: 1953, Astron. J. 58, 61.

Sandage, A.: 1958, Astrophys. J. 127, 513.

Sandage, A.: 1961, Astrophys. J. 133, 355.

Sandage, A.: 1962, in G. C. McVittie (ed.), 'Problems of Extra-Galactic Research', IAU Symp. $15,359$.

Sandage, A. : 1968a, in L. Woltjer (ed.), Galaxies and the Universe, Columbia University Press, New York, p. 75.

Sandage, A.: 1968b, Astrophys. J. Letters 152, L149.

Sandage, A.: 1968c, Observatory 88, 91.

Sandage, A.: 1970, Astrophys. J. 162, 841.

Sandage, A.: 1971a, Astrophys. J. 166, 13.

Sandage, A.: 1971b, in D. J. K. O'Connell (ed.), Nuclei of Galaxies, North-Holland Publ. Co., Amsterdam, p. 601.

Sandage, A.: 1972a, Astrophys. J. 178, 1.

Sandage, A.: 1972b, Quart. J. Roy. Astron. Soc. 13, 202.

Sandage, A.: 1972c, Quart. J. Roy. Astron. Soc. 13, 282.

Sandage, A. R.: 1972d, Astrophys. J. 176, 21.

Sandage, A.: 1973, Astrophys. J. 183, 711.

Sandage, A. and Hardy, E.: 1973, Astrophys. J. 183, 743.

Sandage, A. and Tammann, G. A.: 1968, Astrophys. J. 151, 531.

Sandage, A. and Tammann, G. A. : 1969, Astrophys. J. 157, 683.

Sandage, A. and Tammann, G. A.: 1971a, Ann. Report Director Hale Obs. 1969-1970, p. 94.

Sandage, A. and Tammann, G. A. : 1971b, Ann. Report Director Hale Obs. 1970-1971, p. 418.

Sandage, A. and Tammann, G. A.: 1971c, Astrophys. J. 167, 293.

Sandage, A. and Tammann, G. A.: 1974a, Astrophys. J., in press.

Sandage, A. and Tammann, G. A.: 1974b (to be published).

Sandage, A., Tammann, G. A., and Hardy, E. : 1972, Astrophys. J. 172, 253.

Seares, F. H., Kapteyn, J. C., and van Rhijn, P. J.: 1930, Mount Wilson Catalogue of Photographic Magnitudes, Carnegie Institution of Washington, Washington.

Searle, L.: 1973, private communication.

Sérsic, J. L.: 1960, Z. Astrophys. 51, 64.

Shane, C. D. and Wirtanen, C. A.: 1967, Lick Obs. Publ. 22, part I.

Shapley, H and Ames, A.: 1932, Harvard Ann. 88, No. 2. 
Smart, N. C. : 1973, private communication.

Spinrad, H., Gunn, J. E., Taylor, B. J., McClure, R. D., and Young, J. W.: 1971, Astrophys. J. $164,11$.

Stebbins, J., Whitford, A. E., and Johnson, H. L. : 1950, Astrophys. J. 112, 469.

Tammann, G. A.: 1969, Mitt. Astron. Ges., No. 27, 55.

Tammann, G. A.: 1972, Astron. Astrophys. 21, 355.

Tammann, G. A. and Sandage, A.: 1968, Astrophys. J. 151, 825.

Tinsley, B. M.: 1973, Astrophys. J. Letters 184, L41.

Upton, E. K. L. : 1970, Astron. J. 75, 1097.

Upton, E. K. L.: 1971, Astron. J. 76, 117.

Westerlund, B. E. and Wall, J. V.: 1969, Astron. J. 74, 335.

Yilmaz, F. and Tammann, G. A. : 1973, in preparation.

Zel'dovich, Ya. B.: 1964, Astron. Zh. 41, 19.

\section{DISCUSSION}

Roeder: If there is no intergalactic medium, the distance-redshift relation is affected in such a way as to raise the value of $q_{0}$, possibly by as much as 0.4 .

Tammann: I believe the most important effect on the formal value of $q_{0}$ is the luminosity evolution of galaxies, and that it is this effect which determines the negative sign of the correction. But I agree completely with you that a detailed quantitative understanding of all observational effects is needed to derive the true numerical value of $q_{0}$. It could well be that your effect helps to prevent the corrected value of $q_{0}$ from becoming negative.

Zel'dovich: Observing the monotonic decrease of $H_{0}$ in the recent past, one wonders how to extrapolate it to the future. It is very important to set a lower limit to it. This has been done by Pikelner (Astron Zh., in press). He argues that the age of the Galaxy is of the order of $T_{\mathrm{G}}=10$ or $12 \times 10^{9} \mathrm{yr}$ from the abundances of the radioactive elements. If it is typical for all galaxies and if we do not see young galaxies, then this value of $T_{\mathrm{G}}$ cannot be much smaller than $H_{0}^{-1}$. Without going into details, $50 \mathrm{~km} \mathrm{~s}^{-1} \mathrm{Mpc}^{-1}$ is near the lower limit of $H_{0} \gtrsim 50 \mathrm{~km} \mathrm{~s}^{-1} \mathrm{Mpc}^{-1}$.

Abell: (1) It may be reasonable to assume that more luminous galaxies have more luminous brightest globular clusters, but I know of no observations to verify this.

(2) The calibration of the linear sizes of $\mathrm{H}$ II regions in Sc I galaxies, and of the van den Bergh luminosity classes rests ultimately on the measurements of angular sizes of resolved $\mathrm{H}$ II regions in galaxies of independently known distances. In how many spiral galaxies, whose distances are known independently of $\mathrm{H}$ II regions, are the $\mathrm{H}$ il regions large enough to be resolved and measured to significant precision? In fact, is there a single Sc I galaxy whose distance is known independently of $\mathrm{H}$ II regions?

Tammann: As to your first question I shall give a more detailed discussion in the written version of my talk; I believe the evidence is reasonably secure that the brightness of the brightest globular clusters varies with the galaxy size. Our route to $H_{0}$ does not use this dependence; I mentioned it here more as an additional illustration of an important effect, and because at the end of the story it nicely confirms our result.

To your second question I should mention that resolution and measuring accuracy of the largest $\mathrm{H}$ II regions is not much of a problem out to $(m-M) \sim 32^{m}$. However, the calibration of their linear sizes depends on only eleven nearby galaxies (in the Local Group and in the M81 group). I think it is the general problem of the extragalactic distance scale that there are just no additional reliable distances to start with. In our case M101 and its five companions may be added to the number of calibrators, because their distance can be derived without using $\mathrm{H}$ II regions. M101 being an Sc I galaxy implies then that we have one independent distance to a supergiant spiral. However, the finally adopted, mean absolute magnitude of Sc I's does not rest on this single value, but is supported by several cross-correlations; for instance the relative luminosities of different luminosity classes can be determined without knowledge of $H_{0}$ and without the use of $\mathrm{H}$ II regions.

Wagoner: I would like to remind everyone that the meanings of $H_{0}$ and $q_{0}$ depend only on well-verified theoretical assumptions but quantities which are often related to them, such as the age of the Universe, depend on assumptions at a more tenuous theoretical level. 